\title{
Study of generalized networks using graph theory
}

\author{
Tomáš Vetrík (iD \\ Department of Mathematics and Applied Mathematics, University of the Free State, Bloemfontein, South \\ Africa
}

\begin{abstract}
We study eccentricity-based indices such as Zagreb eccentricity indices, general eccentricity index and total eccentricity index. We present exact values of eccentricity-based indices of generalized networks related to binary and $m$-ary trees, where $m \geq 2$.
\end{abstract}

Mathematics Subject Classification (2020). 05C90, 05C12

Keywords. graph theory, eccentricity-based index, network

\section{Introduction}

Let $G$ be a network with vertex set $V(G)$ and edge set $E(G)$. The distance between two vertices is the number of edges in a shortest path connecting them. The eccentricity $\operatorname{ecc}_{G}(v)$ of a vertex $v$ is the distance between $v$ and a vertex furthest from $v$ in $G$.

Gao et al. [3] and Imran et al. [5] studied networks $H T(k)$ (see Figure 1) and $S T_{k}^{1}$ (see Figure 2). We generalize those networks.

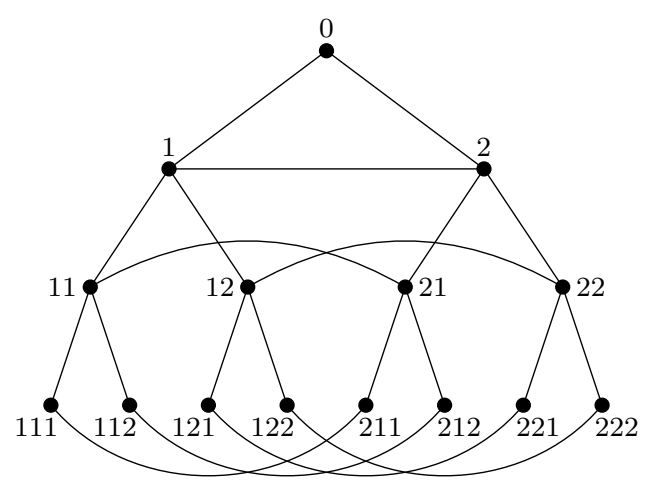

Figure 1. Network $H T(k)$ for $k=3$.

We introduce the sets $H T_{m, k}$ and $S T_{m, k}$ for $m \geq 2$. The special cases for $m=2$ contain the networks $H T(k)$ and $S T_{k}^{1}$ studied in [3] and [5].

We define general networks using complete $m$-ary trees $T_{m, k}$ of height $k \geq 1$. The tree $T_{m, k}$ contains $1+m+m^{2}+\cdots+m^{k}$ vertices which are divided into $k+1$ sets $V_{i}$ for

Email address: vetrikt@ufs.ac.za

Received: 09.11.2020; Accepted: 01.06.2021 


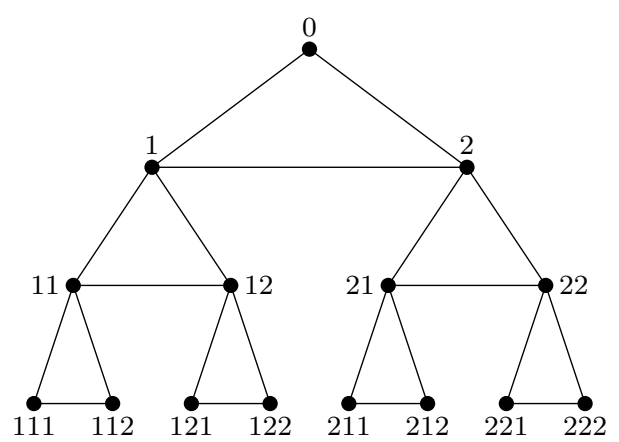

Figure 2. Network $S T_{k}^{1}$ for $k=3$.

$i=0,1,2, \ldots, k$, where $\left|V_{i}\right|=m^{i}$. The vertex in $V_{0}$ is called the root and we denote it by 0 . For $i=1,2, \ldots, k$, we denote the vertices in $V_{i}$ by $\left(x_{1}, x_{2}, \ldots, x_{i}\right)$ or simply $x_{1} x_{2} \ldots x_{i}$, where $x_{1}, x_{2}, \ldots, x_{i} \in\{1,2, \ldots, m\}$. So

$$
V_{i}=\left\{\left(x_{1}, x_{2}, \ldots, x_{i}\right) \mid 1 \leq x_{j} \leq m ; j=1,2, \ldots, i\right\} .
$$

The root is adjacent to all the $m$ vertices $(1),(2), \ldots,(m)$ in $V_{1}$. For $i=1,2, \ldots, k-1$, any vertex $\left(x_{1}, x_{2}, \ldots, x_{i}\right)$ of $V_{i}$ is adjacent to $m$ vertices

$$
\left(x_{1}, x_{2}, \ldots, x_{i}, 1\right),\left(x_{1}, x_{2}, \ldots, x_{i}, 2\right), \ldots,\left(x_{1}, x_{2}, \ldots, x_{i}, m\right)
$$

of $V_{i+1}$. If $m=2$, then $T_{2, k}$ is called the complete binary tree.

The set $S T_{m, k}$ contains all the networks obtained from $T_{m, k}$ by connecting each vertex $v$ in $V_{i}$ for $i=2,3, \ldots, k$, to any $m-1$ vertices in $V_{i}$ whose first coordinate is equal to the first coordinate of $v$, and by connecting every two different vertices in $V_{1}$. One of the networks of $S T_{m, k}$ for $m=3$ and $k=3$ is presented in Figure 3. Note that if $k=1$ or 2, there is only one network in $S T_{m, k}$ for every $m \geq 2$.

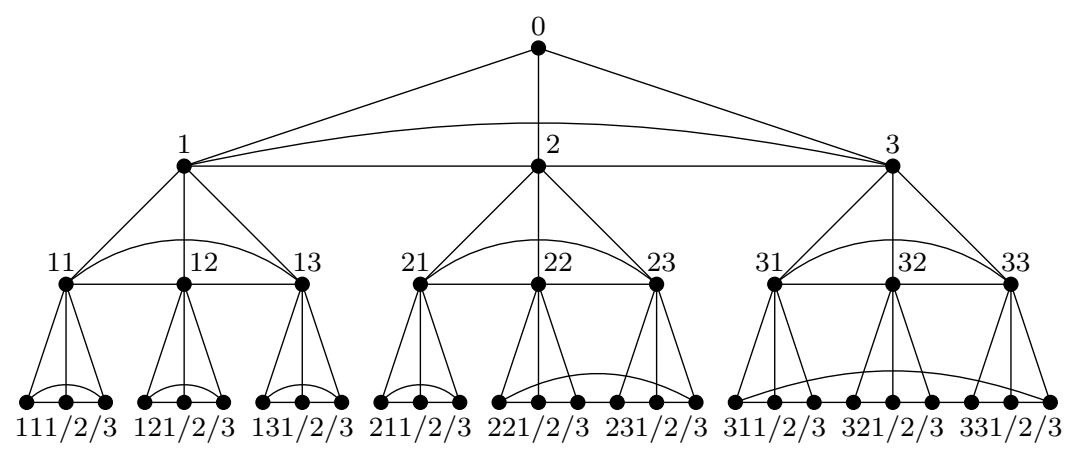

Figure 3. One of the networks in $S T_{m, k}$ for $m=3$ and $k=3$.

For $G \in S T_{m, k}$, no vertex of $V(G) \backslash\left(V_{0} \cup V_{1}\right)$ with the first coordinate $j$ is adjacent to a vertex with the first coordinate $l$, where $j \neq l$. Therefore, the eccentricity of $\left(x_{1}, x_{2}, \ldots, x_{i}\right) \in V_{i}$ for $i \geq 2$ is $k+i-1$. A vertex furthest from $\left(x_{1}, x_{2}, \ldots, x_{i}\right)$ is any vertex $\left(y_{1}, y_{2}, \ldots, y_{k}\right) \in V_{k}$, where $x_{1} \neq y_{1}$ and the shortest path between $\left(x_{1}, x_{2}, \ldots, x_{i}\right)$ and $\left(y_{1}, y_{2}, \ldots, y_{k}\right)$ is

$$
\left(x_{1}, x_{2}, \ldots, x_{i}\right),\left(x_{1}, x_{2}, \ldots, x_{i-1}\right), \ldots,\left(x_{1}\right),\left(y_{1}\right),\left(y_{1}, y_{2}\right), \ldots,\left(y_{1}, y_{2}, \ldots, y_{k}\right) .
$$

The eccentricity of any vertex in $V_{0} \cup V_{1}$ is $k$.

The network $S T_{k}^{1}$ is one of the networks in $S T_{2, k}$. The network $S T_{k}^{1}$ is obtained from the complete binary tree $T_{2, k}$ by joining the vertex $\left(x_{1}, x_{2}, \ldots, x_{i-1}, 1\right)$ and the vertex $\left(x_{1}, x_{2}, \ldots, x_{i-1}, 2\right)$ for each $i=1,2, \ldots, k$ and $x_{1}, x_{2}, \ldots, x_{i-1} \in\{1,2\}$. 
The set $H T_{m, k}$ contains all the networks obtained from $T_{m, k}$ by connecting each vertex $v$ in $V_{i}$ for $i=2,3, \ldots, k$, to any $m-1$ vertices in $V_{i}$ whose second coordinate is equal to the second coordinate of $v$, and by connecting any two vertices in $V_{1}$. If $k=1$ or 2 , there is only one network in $H T_{m, k}$ for every $m \geq 2$. The network which is in the set $H T_{m, k}$ if $m=3$ and $k=2$ is presented in Figure 4 .

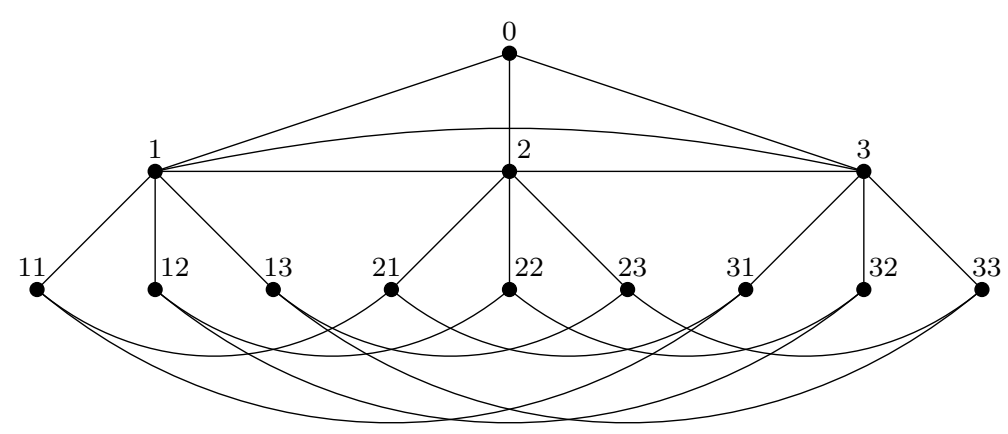

Figure 4. The network which is in the set $H T_{m, k}$ if $m=3$ and $k=2$.

For $G \in H T_{m, k}$, no vertex of $V(G) \backslash\left(V_{0} \cup V_{1}\right)$ with the second coordinate $j$ is adjacent to a vertex with the second coordinate $l$, where $j \neq l$. The eccentricity of $\left(x_{1}, x_{2}, \ldots, x_{i}\right) \in V_{i}$ for $i \geq 2$ is $k+i-1$ A vertex furthest from $\left(x_{1}, x_{2}, \ldots, x_{i}\right)$ is any vertex $\left(y_{1}, y_{2}, \ldots, y_{k}\right) \in V_{k}$, where $x_{1} \neq y_{1}$ and $x_{2} \neq y_{2}$. A shortest path between $\left(x_{1}, x_{2}, \ldots, x_{i}\right)$ and $\left(y_{1}, y_{2}, \ldots, y_{k}\right)$ is for example the path

$$
\left(x_{1}, x_{2}, \ldots, x_{i}\right),\left(x_{1}, x_{2}, \ldots, x_{i-1}\right), \ldots,\left(x_{1}\right),\left(y_{1}\right),\left(y_{1}, y_{2}\right), \ldots,\left(y_{1}, y_{2}, \ldots, y_{k}\right),
$$

where $x_{1} \neq y_{1}$ and $x_{2} \neq y_{2}$. The eccentricity of any vertex in $V_{0} \cup V_{1}$ is $k$. This implies that any network in $S T_{m, k}$ and any network in $H T_{m, k}$ have the same number of vertices of given eccentricity.

The network $H T(k)$ studied in [3] and [5] is one of the networks in $H T_{2, k}$. The network $H T(k)$ is obtained from the complete binary tree $T_{2, k}$ by joining the vertex $\left(1, x_{2}, \ldots, x_{i}\right)$ and the vertex $\left(2, x_{2}, \ldots, x_{i}\right)$ for each $i=1,2, \ldots, k$ and $x_{2}, \ldots, x_{i} \in\{1,2\}$.

Zagreb indices belong to the most well-known topological indices. We mention them in order to present the similarity between the definitions of classical Zagreb indices and Zagreb eccentricity indices. The first Zagreb index $M_{1}$ and the second Zagreb index $M_{2}$ of a network $G$ are defined as

$$
M_{1}(G)=\sum_{v \in V(G)}\left(\operatorname{deg}_{G}(v)\right)^{2}=\sum_{u v \in E(G)}\left(\operatorname{deg}_{G}(u)+\operatorname{deg}_{G}(v)\right)
$$

and

$$
M_{2}(G)=\sum_{u v \in E(G)} \operatorname{deg}_{G}(u) \operatorname{deg}_{G}(v)
$$

where $\operatorname{deg}_{G}(u)$ and $\operatorname{deg}_{G}(v)$ are the degrees of vertices $u$ and $v$ in $G$, respectively. The Zagreb eccentricity indices are defined as

$$
\begin{aligned}
& \xi_{1}(G)=\sum_{v \in V(G)}\left(e c c_{G}(v)\right)^{2}, \\
& \xi_{1}^{\prime}(G)=\sum_{u v \in E(G)}\left(e c c_{G}(u)+e c c_{G}(v)\right), \\
& \xi_{2}(G)=\sum_{u v \in E(G)} \operatorname{ecc}_{G}(u) e c c_{G}(v) .
\end{aligned}
$$


The first Zagreb eccentricity index $\xi_{1}$ and the second Zagreb eccentricity index $\xi_{2}$ were introduced in [7]. The $\xi_{1}^{\prime}$ index was introduced in [4] and we call it the modified Zagreb eccentricity index.

We generalize $\xi_{1}(G)$ and introduce the general eccentricity index

$$
E I_{a}(G)=\sum_{v \in V(G)}\left(e c c_{G}(v)\right)^{a}
$$

for any real number $a$. If $a=1$, we obtain the total eccentricity index

$$
E I_{1}(G)=\sum_{v \in V(G)} e c c_{G}(v)
$$

and $E I_{2}$ is the first Zagreb eccentricity index $\xi_{1}$.

Eccentricity based indices have been studied because of their extensive applications. Eccentricity based indices of nanostar dendrimers were investigated in [2] and other related indices for example in [1] and [6].

\section{Results}

First we compute $\sum_{i=1}^{k} m^{i}, \sum_{i=1}^{k} i m^{i}$ and $\sum_{i=1}^{k} i^{2} m^{i}$ for $m \geq 2$ and $k \geq 1$. Since

$$
\sum_{i=1}^{k} m^{i}=\frac{m\left(m^{k}-1\right)}{m-1},
$$

we obtain

$$
(1-m) \sum_{i=1}^{k} i m^{i}=m+m^{2}+m^{3}+\cdots+m^{k}-k m^{k+1}=\frac{m\left(1-m^{k}\right)}{1-m}-k m^{k+1}
$$

and

$$
\sum_{i=1}^{k} i m^{i}=\frac{m\left(1-m^{k}\right)}{(1-m)^{2}}+\frac{k m^{k+1}}{m-1}=\frac{k m^{k+2}-(k+1) m^{k+1}+m}{(m-1)^{2}} .
$$

We have $(m-1)^{2} \sum_{i=1}^{k} i^{2} m^{i}$

$$
\begin{aligned}
& =m+2 m^{2}+2 m^{3}+2 m^{4}+\cdots+2 m^{k}-\left(k^{2}+2 k-1\right) m^{k+1}+k^{2} m^{k+2} \\
& =m+2 m^{2}\left(1+m+m^{2}+\cdots+m^{k-2}\right)-\left(k^{2}+2 k-1\right) m^{k+1}+k^{2} m^{k+2} \\
& =\frac{2 m^{2}\left(m^{k-1}-1\right)}{m-1}+m-\left(k^{2}+2 k-1\right) m^{k+1}+k^{2} m^{k+2},
\end{aligned}
$$

thus

$$
\begin{aligned}
\sum_{i=1}^{k} i^{2} m^{i} & =\frac{2 m^{k+1}-2 m^{2}}{(m-1)^{3}}+\frac{k^{2} m^{k+2}-\left(k^{2}+2 k-1\right) m^{k+1}+m}{(m-1)^{2}} \\
& =\frac{k^{2} m^{k+3}-\left(2 k^{2}+2 k-1\right) m^{k+2}+(k+1)^{2} m^{k+1}-m^{2}-m}{(m-1)^{3}} .
\end{aligned}
$$

A vertex $v$ of any network in $S T_{m, k}$ and the same vertex $v$ of any network in $H T_{m, k}$ have the same eccentricities, therefore for every network in $S T_{m, k}$ and every network in $H T_{m, k}$, we obtain the same value of the $E I_{a}$ index (note that $a \in \mathbb{R}$ ).

Theorem 2.1. For any network $G \in S T_{m, k} \cup H T_{m, k}$, we have

$$
E I_{a}(G)=k^{a}+\sum_{i=1}^{k} m^{i}(k+i-1)^{a} .
$$


Proof. The network $G$ contains $1+m+m^{2}+\cdots+m^{k}$ vertices. For $i=0,1,2, \ldots, k$, we have $\left|V_{i}\right|=m^{i}$, where $V_{i}$ is the set of vertices at distance $i$ from the root 0 . For $i=1,2, \ldots, k$, any vertex in $V_{i}$ has eccentricity $k+i-1$. The vertex in $0 \in V_{0}$ has eccentricity $k$. Thus

$$
\begin{aligned}
E I_{a}(G) & =\sum_{v \in V_{0}} k^{a}+\sum_{v \in V_{1}} k^{a}+\sum_{v \in V_{2}}(k+1)^{a}+\cdots+\sum_{v \in V_{k}}(2 k-1)^{a} \\
& =m^{0} k^{a}+m^{1} k^{a}+m^{2}(k+1)^{a}+\cdots+m^{k}(2 k-1)^{a} \\
& =k^{a}+\sum_{i=1}^{k} m^{i}(k+i-1)^{a}
\end{aligned}
$$

In Theorems 2.2 and 2.3, we investigate the total eccentricity index $E I_{1}$ and the first Zagreb eccentricity index $E I_{2}\left(=\xi_{1}\right)$ of biological networks, respectively.

Theorem 2.2. For any network $G \in S T_{m, k} \cup H T_{m, k}$, we have

$$
E I_{1}(G)=\frac{(2 k-1) m^{k+2}-2 k m^{k+1}+m^{2}-k m+k}{(m-1)^{2}} .
$$

Proof. By Theorem 2.1, we have

$$
E I_{1}(G)=k+\sum_{i=1}^{k} m^{i}(k+i-1) .
$$

So

$$
\begin{aligned}
E I_{1}(G) & =k+(k-1) \sum_{i=1}^{k} m^{i}+\sum_{i=1}^{k} i m^{i} \\
& =k+(k-1) \frac{m\left(m^{k}-1\right)}{m-1}+\frac{k m^{k+2}-(k+1) m^{k+1}+m}{(m-1)^{2}} \\
& =\frac{(2 k-1) m^{k+2}-2 k m^{k+1}+m^{2}-k m+k}{(m-1)^{2}} .
\end{aligned}
$$

Theorem 2.3. For any network $G \in S T_{m, k} \cup H T_{m, k}$, we have

$$
\begin{aligned}
\xi_{1}(G)= & \frac{(2 k-1)^{2} m^{k+3}-\left(8 k^{2}-4 k-1\right) m^{k+2}+4 k^{2} m^{k+1}}{(m-1)^{3}} \\
& +\frac{(2 k-1) m^{3}-(k+1)^{2} m^{2}+2 k^{2} m-k^{2}}{(m-1)^{3}} .
\end{aligned}
$$

Proof. By Theorem 2.1, we have

$$
E I_{2}=\xi_{1}(G)=k^{2}+\sum_{i=1}^{k} m^{i}(k+i-1)^{2} .
$$

So

$$
\begin{aligned}
\xi_{1}(G) & \left.=k^{2}+\sum_{i=1}^{k} m^{i}\left[(k-1)^{2}+2(k-1) i+i^{2}\right)\right] \\
& =k^{2}+\left(k^{2}-2 k+1\right) \sum_{i=1}^{k} m^{i}+(2 k-2) \sum_{i=1}^{k} i m^{i}+\sum_{i=1}^{k} i^{2} m^{i} \\
& =k^{2}+\left(k^{2}-2 k+1\right) \frac{m\left(m^{k}-1\right)}{m-1}+(2 k-2) \frac{k m^{k+2}-(k+1) m^{k+1}+m}{(m-1)^{2}}
\end{aligned}
$$




$$
\begin{aligned}
& +\frac{k^{2} m^{k+3}-\left(2 k^{2}+2 k-1\right) m^{k+2}+(k+1)^{2} m^{k+1}-m^{2}-m}{(m-1)^{3}} \\
= & \frac{(2 k-1)^{2} m^{k+3}-\left(8 k^{2}-4 k-1\right) m^{k+2}+4 k^{2} m^{k+1}}{(m-1)^{3}} \\
& +\frac{(2 k-1) m^{3}-(k+1)^{2} m^{2}+2 k^{2} m-k^{2}}{(m-1)^{3}} .
\end{aligned}
$$

In Theorem 2.4, we investigate the invariant

$$
I(G)=\sum_{u v \in E(G)} f\left(\operatorname{ecc}_{G}(u), \operatorname{ecc}_{G}(v)\right),
$$

where $f\left(e c c_{G}(u), e c c_{G}(v)\right)$ is a function of $\operatorname{ecc}_{G}(u)$ and $\operatorname{ecc}_{G}(v)$ such that $f\left(\operatorname{ecc}_{G}(u)\right.$, $\left.\operatorname{ecc}_{G}(v)\right)=f\left(\operatorname{ecc}_{G}(v), e c c_{G}(u)\right)$.

If $f\left(\operatorname{ecc}_{G}(u), e c c_{G}(v)\right)=e c c_{G}(u)+e c c_{G}(v)$, we obtain the modified Zagreb eccentricity index If $f\left(\operatorname{ecc}_{G}(u), e c c_{G}(v)\right)=\operatorname{ecc}_{G}(u) e c c_{G}(v)$, we obtain the second Zagreb eccentricity index.

Theorem 2.4. For any network $G \in S T_{m, k} \cup H T_{m, k}$, we have

$$
I(G)=m \cdot f(k, k)+\sum_{i=2}^{k} m^{i} f(k+i-2, k+i-1)+\sum_{i=1}^{k} \frac{m^{i+1}-m^{i}}{2} f(k+i-1, k+i-1) .
$$

Proof. The network $G$ contains

$$
\left(m+m^{2}+\cdots+m^{k}\right)+\left(\frac{(m-1) m}{2}+\frac{(m-1) m^{2}}{2}+\cdots+\frac{(m-1) m^{k}}{2}\right)
$$

edges. Let

$$
E_{i, j}=\left\{u v \in E(G) \mid u \in V_{i}, v \in V_{j}\right\} .
$$

So the set $E_{i, j}$ contains the edges incident with one vertex in $V_{i}$ and the other vertex in $V_{j}$. We obtain

and

$$
\left|E_{0,1}\right|=m,\left|E_{1,2}\right|=m^{2}, \ldots,\left|E_{k-1, k}\right|=m^{k}
$$

Note that

$$
\left|E_{1,1}\right|=\frac{(m-1) m}{2},\left|E_{2,2}\right|=\frac{(m-1) m^{2}}{2}, \ldots,\left|E_{k, k}\right|=\frac{(m-1) m^{k}}{2} .
$$

$$
E(G)=\left(E_{0,1} \cup E_{1,2} \cup \cdots \cup E_{k-1, k}\right) \cup\left(E_{1,1} \cup E_{2,2} \cup \cdots \cup E_{k, k}\right) .
$$

For $i=1,2, \ldots, k$, any vertex in $V_{i}$ has eccentricity $k+i-1$ and the vertex $0 \in V_{0}$ has eccentricity $k$. Thus we obtain

$$
\begin{aligned}
I(G)= & \sum_{u v \in E(G)} f\left(\operatorname{ecc}_{G}(u), \operatorname{ecc}_{G}(v)\right) \\
= & \sum_{u v \in E_{0,1}} f(k, k)+\sum_{u v \in E_{1,2}} f(k, k+1) \\
& +\sum_{u v \in E_{2,3}} f(k+1, k+2)+\cdots+\sum_{u v \in E_{k-1, k}} f(2 k-2,2 k-1) \\
& +\sum_{u v \in E_{1,1}} f(k, k)+\sum_{u v \in E_{2,2}} f(k+1, k+1)+\cdots+\sum_{u v \in E_{k, k}} f(2 k-1,2 k-1) \\
= & m \cdot f(k, k)+m^{2} f(k, k+1) \\
& +m^{3} f(k+1, k+2)+\cdots+m^{k} f(2 k-2,2 k-1)+\frac{(m-1) m}{2} f(k, k)
\end{aligned}
$$




$$
\begin{aligned}
& +\frac{(m-1) m^{2}}{2} f(k+1, k+1)+\cdots+\frac{(m-1) m^{k}}{2} f(2 k-1,2 k-1) \\
= & m \cdot f(k, k)+\sum_{i=2}^{k} m^{i} f(k+i-2, k+i-1) \\
& +\sum_{i=1}^{k} \frac{m^{i+1}-m^{i}}{2} f(k+i-1, k+i-1) .
\end{aligned}
$$

In Theorem 2.5, we obtain the values of the modified Zagreb eccentricity index $\xi_{1}^{\prime}$ and the second Zagreb eccentricity index $\xi_{2}$ of the generalized networks.

Theorem 2.5. For any network $G \in S T_{m, k} \cup H T_{m, k}$, we have

$$
\xi_{1}^{\prime}(G)=\frac{(2 k-1) m^{k+3}-2 m^{k+2}-(2 k-1) m^{k+1}-(k-2) m^{3}+k m}{(m-1)^{2}}
$$

and

$$
\begin{aligned}
\xi_{2}(G)= & k m+\left[\frac{m+1}{2}\left(k^{2}-2 k+1\right)-k+1\right] \frac{m^{k+1}-m}{m-1} \\
& +[(m+1)(k-1)-1] \frac{k m^{k+2}-(k+1) m^{k+1}+m}{(m-1)^{2}} \\
& +\left(\frac{m+1}{2}\right) \frac{k^{2} m^{k+3}-\left(2 k^{2}+2 k-1\right) m^{k+2}+(k+1)^{2} m^{k+1}-m^{2}-m}{(m-1)^{3}} .
\end{aligned}
$$

Proof. For the $\xi_{1}^{\prime}$ index, we get $f\left(\operatorname{ecc}_{G}(u), e c c_{G}(v)\right)=e c c_{G}(u)+e c c_{G}(v)$, so $f(k+i-$ $2, k+i-1)=2 k+2 i-3$ and $f(k+i-1, k+i-1)=2 k+2 i-2$. Then by Theorem 2.4,

$$
\begin{aligned}
\xi_{1}^{\prime}(G) & =2 k m+\sum_{i=2}^{k} m^{i}(2 k+2 i-3)+\sum_{i=1}^{k} \frac{m^{i+1}-m^{i}}{2}(2 k+2 i-2) \\
& =2 k m-(2 k-1) m+\sum_{i=1}^{k} m^{i}(2 k+2 i-3)+\sum_{i=1}^{k}(m-1) m^{i}(k+i-1) \\
& =m+[(k-1)(m-1)+2 k-3] \sum_{i=1}^{k} m^{i}+(m+1) \sum_{i=1}^{k} i m^{i} \\
& =m+[(k-1) m+k-2] \frac{m\left(m^{k}-1\right)}{m-1}+(m+1) \frac{k m^{k+2}-(k+1) m^{k+1}+m}{(m-1)^{2}} \\
& =\frac{(2 k-1) m^{k+3}-2 m^{k+2}-(2 k-1) m^{k+1}-(k-2) m^{3}+k m}{(m-1)^{2}} .
\end{aligned}
$$

For the $\xi_{2}$ index, we get $f\left(\operatorname{ecc}_{G}(u), \operatorname{ecc}_{G}(v)\right)=\operatorname{ecc}_{G}(u) e c c_{G}(v)$, so

$$
f(k+i-2, k+i-1)=(k+i-2)(k+i-1)=\left(k^{2}-3 k+2\right)+(2 k i-3 i)+i^{2}
$$

and

$$
f(k+i-1, k+i-1)=(k+i-1)(k+i-1)=\left(k^{2}-2 k+1\right)+(2 k i-2 i)+i^{2}
$$

Then by Thworem 2.4,

$$
\begin{aligned}
\xi_{2}(G)= & k^{2} m+\sum_{i=2}^{k} m^{i}\left[\left(k^{2}-3 k+2\right)+(2 k i-3 i)+i^{2}\right] \\
& +\sum_{i=1}^{k} \frac{m^{i+1}-m^{i}}{2}\left[\left(k^{2}-2 k+1\right)+(2 k i-2 i)+i^{2}\right]
\end{aligned}
$$




$$
\begin{aligned}
= & k^{2} m-k(k-1) m+\sum_{i=1}^{k} m^{i}\left[\left(k^{2}-3 k+2\right)+(2 k i-3 i)+i^{2}\right] \\
& +\sum_{i=1}^{k} \frac{(m-1) m^{i}}{2}\left[\left(k^{2}-2 k+1\right)+(2 k i-2 i)+i^{2}\right] \\
= & k m+\left[\frac{m-1}{2}\left(k^{2}-2 k+1\right)+\left(k^{2}-3 k+2\right)\right] \sum_{i=1}^{k} m^{i} \\
& +[(m-1)(k-1)+(2 k-3)] \sum_{i=1}^{k} i m^{i}+\left(\frac{m-1}{2}+1\right) \sum_{i=1}^{k} i^{2} m^{i} \\
= & k m+\left[\frac{m+1}{2}\left(k^{2}-2 k+1\right)-k+1\right] \frac{m^{k+1}-m}{m-1} \\
& +[(m+1)(k-1)-1] \frac{k m^{k+2}-(k+1) m^{k+1}+m}{(m-1)^{2}} \\
& +\left(\frac{m+1}{2}\right) \frac{k^{2} m^{k+3}-\left(2 k^{2}+2 k-1\right) m^{k+2}+(k+1)^{2} m^{k+1}-m^{2}-m}{(m-1)^{3}} .
\end{aligned}
$$

The following corollary holds for any network in biological networks in which one person transmits a virus or bacteria to two other people, so it holds also for the networks $S T_{k}^{1}$ and $H T(k)$.

Corollary 2.6. For any network $G \in S T_{2, k} \cup H T_{2, k}$, we have

$$
\xi_{1}^{\prime}(G)=(k-1) 2^{k+4}-k 2^{k+2}+2^{k+1}-6 k+16
$$

and

$$
\xi_{2}(G)=(1-k) 2^{k+4}+k(k-1) 2^{k+3}+\left(k^{2}-k+1\right) 2^{k+2}+2^{k+1}-3 k^{2}+16 k-22 .
$$

Proof. By Theorem 2.5, the modified Zagreb eccentricity index

$$
\begin{aligned}
\xi_{1}^{\prime}(G) & =(2 k-1) 2^{k+3}-2 \cdot 2^{k+2}-(2 k-1) 2^{k+1}-(k-2) 2^{3}+3 k \\
& =(k-1) 2^{k+4}-k 2^{k+2}+2^{k+1}-6 k+16
\end{aligned}
$$

and the second Zagreb eccentricity index

$$
\begin{aligned}
\xi_{2}(G)= & 2 k+\left[\frac{3}{2}\left(k^{2}-2 k+1\right)-k+1\right]\left(2^{k+1}-2\right) \\
& +[3(k-1)-1]\left[k 2^{k+2}-(k+1) 2^{k+1}+2\right] \\
& +\frac{3}{2}\left[k^{2} 2^{k+3}-\left(2 k^{2}+2 k-1\right) 2^{k+2}+(k+1)^{2} 2^{k+1}-2^{2}-2\right] \\
= & (1-k) 2^{k+4}+k(k-1) 2^{k+3}+\left(k^{2}-k+1\right) 2^{k+2}+2^{k+1}-3 k^{2}+16 k-22 .
\end{aligned}
$$

It is easy to check that for $G \in S T_{2, k} \cup H T_{2, k}$, we obtain $\xi_{1}^{\prime}(G)=6$ and $\xi_{2}(G)=3$ if $k=1$ and $\xi_{1}^{\prime}(G)=44$ and $\xi_{2}(G)=54$ if $k=2$. These values can be computed after drawing the networks of the set $S T_{2, k} \cup H T_{2, k}$. The only network in $S T_{2, k} \cup H T_{2, k}$ for $k=1$ is the triangle. The only networks in the set $S T_{2, k} \cup H T_{2, k}$ for $k=2$ are the networks $S T_{2}^{1}$ and $H T(2)$.

Acknowledgment. This work is based on the research supported by the National Research Foundation of South Africa (Grant Number 129252). 


\section{References}

[1] F. Asif, Z. Zahid, S. Zafar, M.R. Farahani and W. Gao, On topological properties of some convex polytopes by using line operator on their subdivisions, Hacet. J. Math. Stat. 49 (1), 136-146, 2020.

[2] R. Farooq and M.A. Malik, On some eccentricity based topological indices of nanostar dendrimers, Optoelectronics and Advanced Materials, Rapid Communications 9 (56), 842-849, 2015.

[3] W. Gao, H. Wu, M.K. Siddiqui and A.Q. Baig, Study of biological networks using graph theory, Saudi J. Biol. Sci. 25 (6), 1212-1219, 2018.

[4] M. Ghorbani and M.A. Hosseinzade, A new version of Zagreb indices, Filomat 26 (1), 93-100, 2012.

[5] M. Imran, M.A. Iqbal, Y. Liu, A.Q. Baig, W. Khalid and M.A. Zaighum, Computing eccentricity-based topological indices of 2-power interconnection networks, J. Chem. 2020, 3794592, 2020.

[6] M.K. Siddiqui, M. Imran and M. Saeed, Topological properties of face-centred cubic lattice, Hacet. J. Math. Stat. 49 (1), 195-207, 2020.

[7] D. Vukičević and A. Graovac, Note on the comparison of the first and second normalized Zagreb eccentricity indices, Acta Chim. Slov. 57 (3), 524-528, 2010. 